\title{
Viral infections in goose flocks in Poland
}

\author{
W. Kozdruń, G. Woźniakowski, E. Samorek-Salamonowicz, H. Czekaj \\ National Veterinary Research Institute, Department of Poultry Viral Diseases, \\ Partyzantów 57 Avenue, 24-100 Pulawy, Poland
}

\begin{abstract}
The aim of this study was to determine the infectious agents isolated from infection - suspected geese sent for the diagnostic examination to National Veterinary Research Institute. The birds were sent from goose flocks localized in different parts of Poland. Totally, 1,013 birds from 122 flocks were examined. The presence of goose parvovirus (GPV), goose haemorrhagic polyomavirus (GHPV), and goose circovirus $(\mathrm{GoCV})$ was detected by triplex PCR. The presence of GPV DNA was shown in 36 flocks. The disease was most frequently diagnosed in goslings aging 3.5 weeks (ten flocks), and 2.5 weeks (six flocks). The analysis of the nucleotide sequence of VP1 encoding region has shown close similarity of Polish GPV strains within the group which ranged from $92 \%$ to $100 \%$. Moreover, the similarity level of these strains with GPV isolated in Europe was from $91.3 \%$ to $100 \%$. The occurrence of GoCV DNA was shown in 25 goose flocks. The presence of GoCV DNA was found among geese aged from 2 to 6 weeks, but predominantly in those aging 3.5 (three flocks) and 5 weeks (five flocks). The sequence analysis of PCR products from the sequenced region of ORFC1 capsid protein of GoCV has shown that Polish isolates share from $85 \%$ to $91 \%$ similarity with the sequences of GoCV strains isolated in other countries. The presence of DNA of GHPV was found in 3-week-old geese. During the last 2 years the presence of GHPV was confirmed in three flocks of goslings at the age from 3 to 3.5 weeks. During the last 12 years the occurrence of co-infection with GPV and GoCV was detected in six flocks aging from 5 to 6 weeks.
\end{abstract}

Key words: geese, parvovirus, circovirus, polyomavirus, infection

\section{Introduction}

Infectious diseases of viral aetiology present an epidemic threat in goose production. Derzsy's disease is the most important viral disease of geese but infections with polyoma- and circoviruses are frequent as well.

Derzsy's disease (DD) is caused by Derzsy's disease virus (DDV) also called goose parvovirus (GPV). The birds susceptible to the infection are domestic and wild geese as well as Mullard and Barbarie ducks. The course of the infection and specific clinical symp- toms depend on the age of birds, their immune status, and breeding conditions (Brown et al. 1995).

In spite of protective vaccination that limited the occurrence of Derzsy's disease, it is still a cause of considerable losses in goose flocks. The morbidity due to DD reach up to $80 \%$ of birds in a flock while the mortality is frequently about $60 \%$.

The first cases of the disease were reported in Poland in the 1970s. Due to this fact, protective vaccinations of breeder flocks were introduced in 1982, what remarkably limited the occurrence of the disease. However, in the middle of the1990s, the number 
of clinical cases of DD increased again. Actually, the disease progresses as a chronic form with lower mortality than before. In Poland the disease is responsible for $16.5 \%$ to $19 \%$ of overall geese mortality. These data indicate the importance of this problem. Another important infectious disease of geese is syndrome of hemorrhagic nephritis and enteritis (HNEG) caused by goose haemorrhagic polyomavirus (GHPV). The disease is frequent in goslings of the age from $4^{\text {th }}$ to $10^{\text {th }}$ weeks (Guerin et al. 2000). The course of HNEG is acute, the mortality is high and the observed lesions in affected birds may give false evidence of DD. The third factor of viral infections of waterfowl is represented by goose circovirus (GoCV). These infections are frequent in goslings older than 5 weeks. The clinical symptoms are non-specific and include weight losses and feathering disorders, which are also observed in case of DD. The main role of this virus is associated with immunosuppression (Todd 2000).

Therefore, in case of mortality occurring in goose flocks, these three viral diseases of geese should be taken into account during differential diagnosis.

The aim of this study was to determine the infectious agents isolated from geese sent for the diagnostic examination to National Veterinary Research Institute during the years 1998-2010.

\section{Materials and Methods}

Birds. The infection-suspected birds were sent to the laboratory during 1998-2010 from goose farms localised in different parts of Poland. Totally, 1,013 birds from 122 flocks were examined. The number of birds in the flocks ranged from 1,200 to 12,000 , most frequently from 3,000 to 5,000 birds. The age of the examined geese ranged from 1 to 9 weeks, but predominantly between 2 and 5 weeks. The clinical symptoms and pathological lesions suggested the occurrence of Derzsy's disease.

Standard viruses. The vaccine strain MFP from Palmivax vaccine (Mérial, France) was used as the standard strain of GPV. The GoCV standard strain and GHPV strain were obtained thanks to courtesy of Dr Vilmos Palya (Ceva-Phylaxia, Ceva Sainte Animale, Budapest, Hungary).

DNA extraction. DNA from standard strains was extracted from $200 \mu$ of viral stock according to recommended procedure. Sections of the liver, heart, and kidneys were collected from the examined birds during post-mortem examination. Next, the sections were homogenised and total DNA was extracted according the procedure recommended by the manufacturer of QIAmp DNA Mini Kit (Qiagen, Germany).
PCR primers. Three pairs of specific primers were used. The sequences of primers used for GPV detection were: VPD1 - 5, CCG GGT TGC AGG AGG TAC 3'; VPD1 - 5' AGC TAC AAC AAC CAC ATC 3'. The expected PCR product size was $806 \mathrm{bp}$. For GoCV the primer sequences were: GCVP1 - 5, TAA ATG CGA GTT TGA TGT GTC T 3'; GCVP2 - 5' CAT TTA ACC CCT TCC AAA GAG T 3', and the expected product size was $571 \mathrm{bp}$, while for GHPV the sequences were as follows: GPOL1 - 5' GAG GTT GTT GGA GTG ACC ACA ATG 3'; GPOL2 - 5' ACA ACG GTG CAA TTC CAA GGG TTC 3' with the expected product about $180 \mathrm{bp}$ long.

Triplex PCR assay. Amplification was carried out in $25 \mu \mathrm{l}$ of reaction mixture that contained: $2.5 \mu \mathrm{l}$ of $10 \mathrm{X}$ concentrated PCR buffer (EurX, Poland), $1 \mu \mathrm{l}$ of dNTP mixture $(0.2 \mathrm{mM}$ of each dNTP), $1 \mu \mathrm{l}$ of $\mathrm{MgSO}_{4}(5 \mathrm{mM}), 2 \mu \mathrm{l}$ of each primer (VPD, GCV, GPOL) (10 mM), $2 \mu$ l of template DNA, $0.5 \mu \mathrm{l}$ of Taq polymerase $(5 \mathrm{U} / \mu \mathrm{l})$, and PCR-grade deionised water. The reaction conditions were as follows: 30 cycles: $94^{\circ} \mathrm{C} / 30 \mathrm{~s}$ (initial denaturation), $52^{\circ} \mathrm{C} / 30 \mathrm{~s}$ (primers annealing), $72^{\circ} \mathrm{C} / 1$ minutes (elongation of products), $72^{\circ} \mathrm{C} / 10$ minutes (final elongation of products). Triplex PCR products were separated in $2 \%$ agarose gel with addition of ethidium bromide $(0.5 \mu \mathrm{g} / \mathrm{ml})$. To each well in gel, $5 \mu \mathrm{l}$ of triplex PCR products and $1 \mu \mathrm{l}$ of loading buffer (Fermentas, Lithuania) were loaded.

Electrophoresis of PCR products. The electrode buffer used for electrophoresis was TBE (Tris - Base $10.8 \mathrm{~g}$, boric acid $5.5 \mathrm{~g}, 4 \mathrm{ml} 0.5 \mathrm{M}$ EDTA, pH 8.0). Electrophoresis was carried out for $1 \mathrm{~h}$ under the voltage of $10 \mathrm{~V} / \mathrm{cm}$ agarose gel. The results were documented using UV-light transiluminator (Vilber-Lourmat, France). Each product was compared with the molecular mass marker of $80-10000 \mathrm{bp}$ (Mass ruller DNA ladder 100 bp plus, Fermentas, Lithuania).

Sequence analysis. After gel electrophoresis the PCR products were cut from the gel and extracted from agarose slices using Gel Extraction Kit (Qiagen, Germany). The purified products were sequenced using the primers used for PCR by the Institute of Biochemistry and Biophysics of the Polish Academy of Science in Warsaw, Poland. The raw sequences were manually edited and compared with sequences accessible in the NCBI GeneBank database using BLAST algorithm.

\section{Results}

During the twelve years (1998-2010) totally 1,013 birds from 122 flocks were examined. According to interviews and our observations, the clinical symptoms 
occurred predominantly in the $1^{\text {st }}$ and $2^{\text {nd }}$ week of life of the birds and were manifested by a decrease in consumption of water and food, locomotor dysfunction, gathering, inhibition of growth, and feathering disorders. Meanwhile, in some of birds breathing difficulties, watery diarrhoea and neural disorders visible as paralysis of legs and neck were observed. The morbidity of the birds in the flock ranged from $30 \%$ to $60 \%$.

The analysis of mortality of the birds in the examined flocks has shown that the mean percentage of dead birds was $13.95 \%$ to $42.45 \%$ and the range was from $9.9 \%$ to $61.7 \%$. The highest mortality of birds was noted in $1999(42.45 \%)$ and the lowest in 2003 $(13.95 \%)$. Our personal observations have shown that after introduction to the market of the inactivated bivalent vaccine containing goose parvovirus and Muscovy duck parvovirus, the proportion of dead birds affected by Derzsy's disease has considerably decreased. Meanwhile, the mortality observed in 2008 was $17.40 \%$ while in 2010 was only $5.70 \%$ (Fig. 1 ).

The clinical symptoms in geese were predominantly observed as weight losses (33\%). Other clinical symptoms and anatomopathological lesions of the

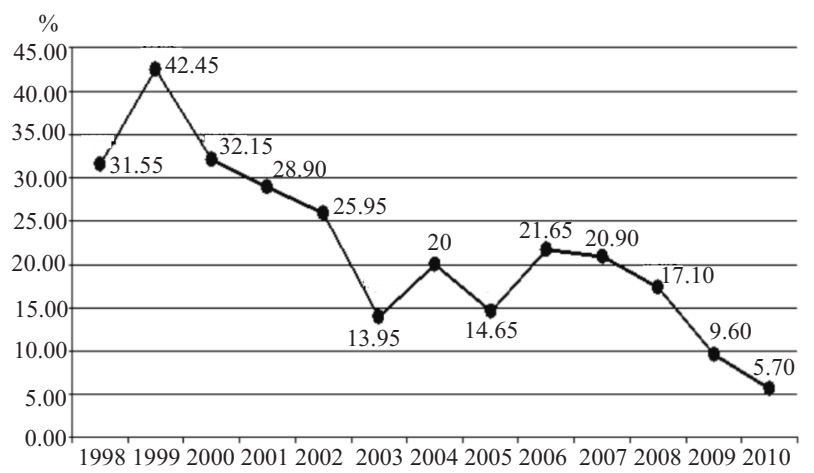

Fig. 1. Mortality of birds in examined flocks during 1998-2010.

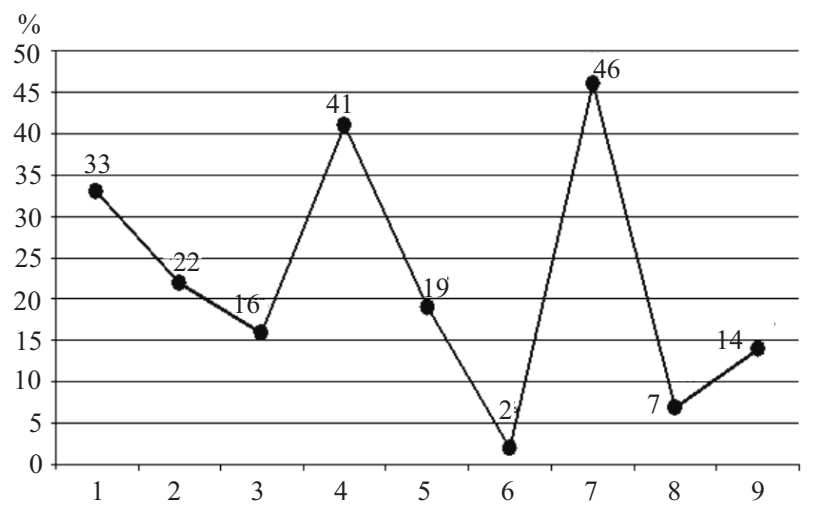

Fig. 2. An occurence of lesions in examined birds. Descriptions: 1 - weight losses; 2 - liver enlargement; 3 - pale liver; 4 -liver congestion; 5 - fibrinous perihepatitis; 6 - necrosis focuses; 7 - rounded heart apex; 8 -fibrinous pericarditis; 9 - congestion of intestines. minor significance are presented in the Fig. 2. The most frequently occurring lesions were observed in the liver and they included congestion $(41 \%)$ or paleness $(16 \%)$, enlargement of the organ $(22 \%)$ and fibrinous perihepatitis $(19 \%)$. Additionally, the occurrence of small necrotic focuses was found in $2 \%$ of birds.

The lesions were also observed in other visceral organs. Rounded heart apex was found in $46 \%$ of the examined birds, $7 \%$ of the birds showed fibrinous pericarditis, while in $14 \%$ of the geese congestion of the intestines was found. Interestingly, both fibrinous pericarditis and congestion of the intestines were observed in birds at the age over 3.5 week.

The study conducted by triplex PCR has shown the presence of GPV DNA in 36 examined flocks (Fig. 3). The number of GPV-positive flocks was constant during the studied period and concerned three - four flocks per year. These flocks originated from areas with the highest density of goose breeding, predominantly from the west and east part of Poland. The disease was most frequently diagnosed in gooslings at the age of 3.5 weeks (ten flocks) and 2.5 weeks (six flocks) (Fig. 4). The analysis of the nucleotide sequence of VP1 encoding region has shown a close similarity of isolated Polish GPV strains which ranged from $92 \%$ to $100 \%$. Moreover, the similarity level of these strains with GPV strains isolated in Europe was from $91.3 \%$ to $100 \%$. These data suggest that Polish GPV strains have the common European origin.

The study on the occurrence of GoCV has shown the presence of the virus in 25 goose flocks. The first case was diagnosed in 2002 and the positive flocks were localised in the west and east part of Poland similarly to GPV localisation. The presence of GoCV DNA was found among geese at the age from 2 to 6 weeks, but predominantly 3.5 (three flocks) and 5 weeks (five flocks) of age (Fig. 5). The sequence analysis of PCR products from the sequenced region of ORFC1 capsid protein of GoCV has shown that Polish isolates share from $85 \%$ to $91 \%$ similarity with the sequences of GoCV strains isolated in other countries.

During the third stage of this study, the presence of DNA of GHPV was found for the first time in 2007 in 3-week-old geese. During 1998-2010 the presence of GHPV was confirmed in three flocks of gooslings aging from 3 to 3.5 weeks. The sequencing of PCR products of GHPV was not conducted in this study; however, so far the occurrence and analysis of GHPV was done in Germany and Hungary, what indicates its importance in epidemiology of viral infection of geese.

The applied triplex PCR allowed for easy DNA detection of three waterfowl viruses. This technique 


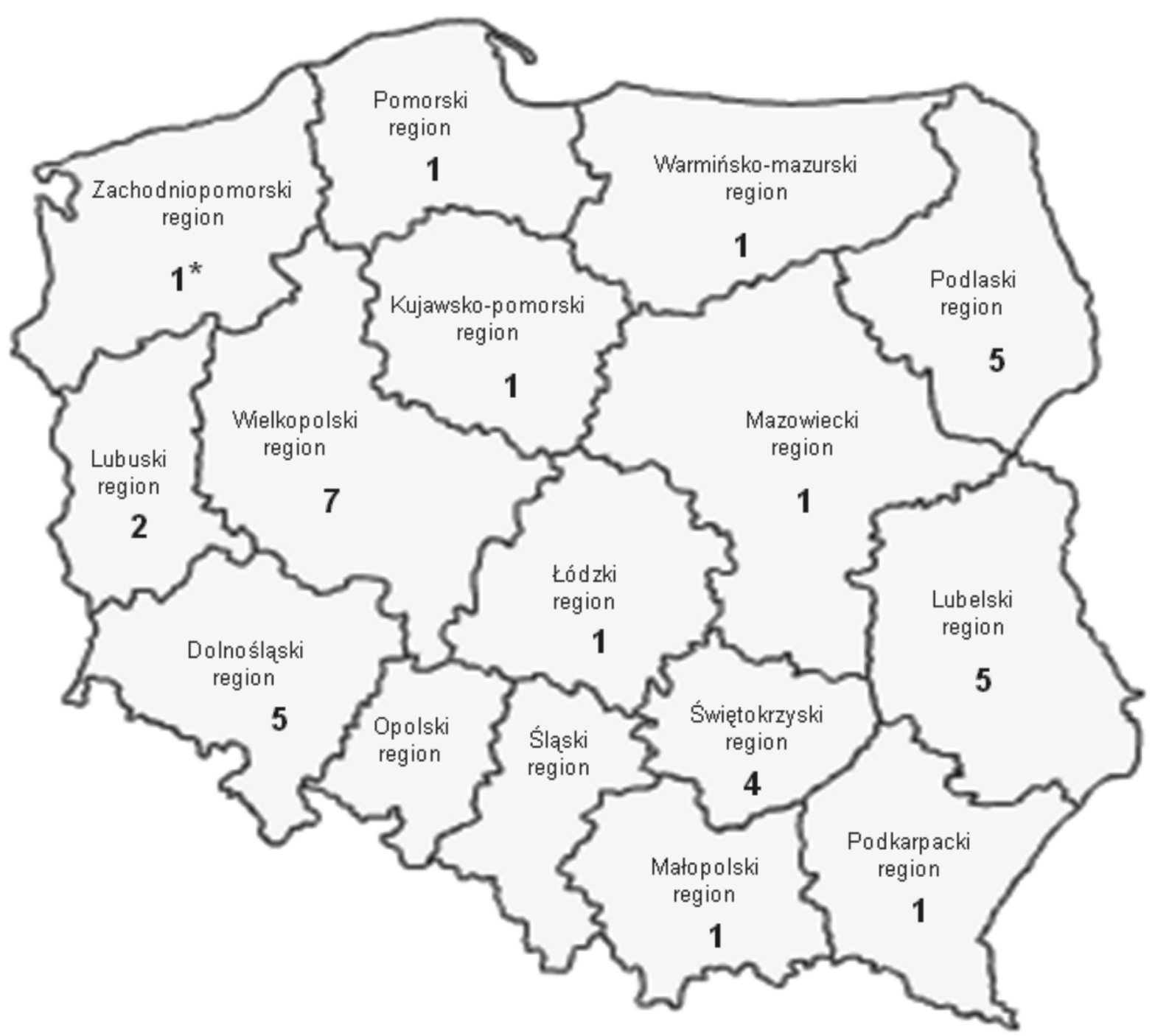

Fig. 3. The Derzsy's disease in Poland during 1998-2010.

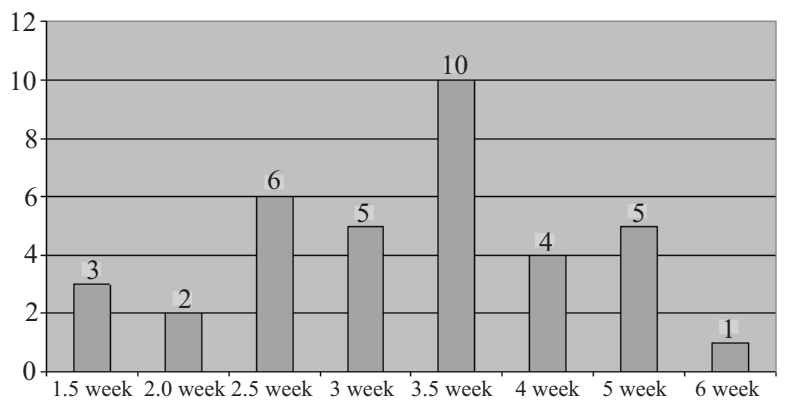

Fig. 4. An occurrence of GPV vs. the age of examined birds 1998-2010.

is useful in differential diagnosis of viral infection of geese, which are frequently manifested by similar clinical symptoms and pathological lesions.

This aspect has a major importance when taking into account that during the last 12 years, in six examined flocks with geese aging from 5 to 6 weeks, the

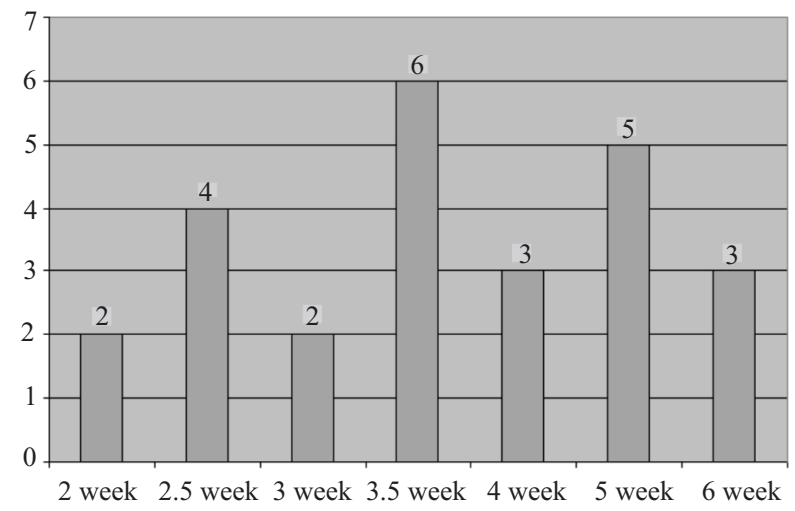

Fig. 5. An occurrence of GoCV vs. the age of examined birds 1998-2010.

occurrence of co-infection with GPV and GoCV was detected (Fig. 6). This may suggest the possible role of these viruses in immunosuppression of the birds, which needs to be studied more comprehensively. 


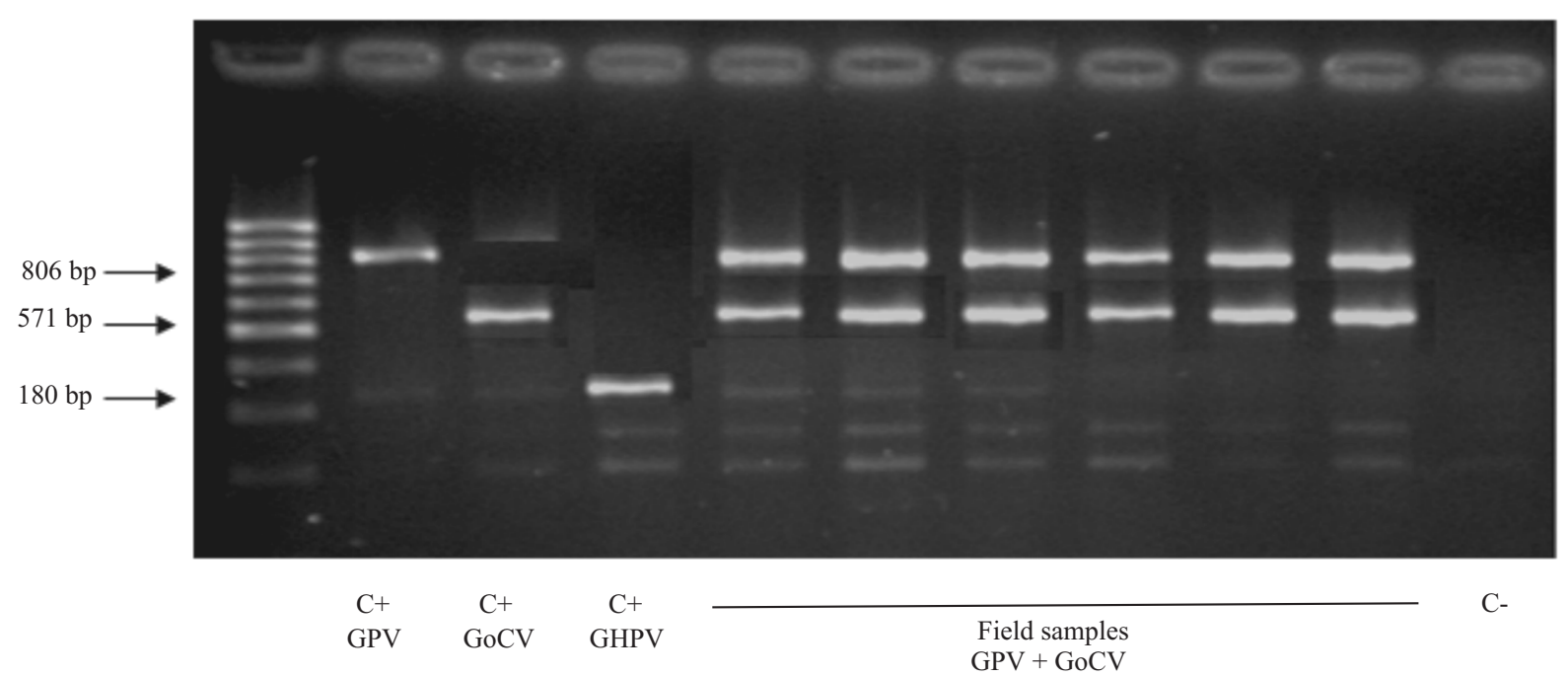

Fig. 6. Triplex PCR. Detection of GPV, GoCV and GHPV in geese. C+ - positive controls, C- - negative control - DNA extracted from non-infected goose fibroblasts (GEF).

\section{Discussion}

The present results are the first analysis of viral infection in flocks of geese in Poland.

The most important viral infection is the goose parvovirus infection. Despite the prophylactic vaccination it is still the cause of deaths of young birds. As reported here, parvovirus infection were found in 36 goose flocks. Goose parvovirus was isolated in many countries of Europe and Asia. In Japan most GPV was isolated in geese at about 2 weeks, and mortality among birds was up $80 \%-100 \%$. GPV infection has also been reported in England and Wales and clinical symptoms and pathological changes were identical to the changes described in geese in Poland. In Sweden mortality of birds due to infection with GPV was from $40 \%$ to $90 \%$ (Takehara et al. 1995, Jansson et al. 2007, Irvine et al. 2008).

The goose circovirus infection was detected in 25 examinated goose flocks. The results of phylogenetic analysis indicated the similarity of Polish isolates with GoCV strains isolated worlwide, but also suggest the variable nature of circoviruses which will be studies in the future (Chen et al. 2003, Ball et al. 2004, Yu et al. 2007).

The goose polyomavirus was detected in 3 examinated goose flocks. The sequencing of PCR products of GHPV was not conducted in this study, however, so far the occurrence and analysis of GHPV was done in Germany and Hungary, what indicates its importance in epidemiology of viral infection of geese (Miksch et al. 2002, Palya et al. 2004).

The obtained results pointed out on the important role of viral disease of geese, which are still an epi- zootic problem. As we presented in our study, in spite of annually applied prophylaxis of GPV infection, the coexistent infectious with GoCV and GHPV make this problem more complexive and indirectly influence the health status of geese.

\section{References}

Ball NW, Smyth JA, Weston JH, Borghmans BJ, Palya V, Glavits R, Ivanics E, Dan A, Todd D (2004) Diagnosis of goose circovirus infection in Hungarian geese samples using polymerase chain reaction and dot blot hybridization tests. Avian Pathol 33: 51-58

Brown KE, Green SW, Young NS (1995) Goose parvovirus - an autonomous member of the dependovirus genus? Virology 210: 283-291.

Chen CL, Chang PC, Lee MS, Shien JH, Ou SJ, Shieh HK (2003) Nucleotide sequences of goose circovirus isolated in Taiwan. Avian Pathol 32: 165-171.

Guerin JL, Gelfi L, Dubois L, Vuillaume A, Boucraut-Baralon C, Pinqret JL (2000) A novel polyomavirus (goose hemorrhagic polyomavirus) is the agent of hemorrhagic nephritis enteritis of geese. J Virol 74: 4523-4529.

Irvine R, Ceeraz V, Cox B, Twomey F, Young S, Bradshaw J, Featherstone C, Holmes JP, Ainsworth H, Jones $\mathrm{R}$ (2008) Goose parvovirus in Great Britain. Vet Rec 163: 461.

Jansson DS, Feinstein R, Kardi V, Mato T, Palya V (2007) Epidemiologic investigation of an outbreak of goose parvovirus infection in Sweden. Avian Dis 51: 609-613.

Miksch K, Grossman E, Kohler K, Johne R (2002) Detection of goose haemorrhagic polyomavirus (GHPV) in flocks with haemorrhagic nephritis and enteritis of geese in southern Germany. Berl Munch Tierarztl Wochenschr 115: 390-394. 
Palya V, Ivanics E, Glavits R, Dan A, Mato T, Zarka $P$ (2004) Epizootic occurrence of haemorrhagic nephritis enteritis virus infection of geese. Avian Pathol 33: 244-250.

Takehara K, Nishio T, Hayashi Y, Kanda J, Sasaki M, Abe N, Hiraizumi M, Saito S, Yamada T, Haritani M (1995) An outbreak of goose parvovirus infection in Japan. J Vet Med Sci 57: 777-779.
Todd D (2000) Circoviruses: immunosuppressive threats to avian species: a review. Avian Pathol 29: 373-394.

Yu X, Zhu C, Zheng X, He S, Liu X (2007) Genome analysis and epidemiological investigation of goose circovirus detected in eastern China. Virus Genes 35: 605-609. 